\title{
SPECTRA OF WOLF-RAYET TYPE CENTRAL STARS AND THEIR ANALYSIS
}

\author{
W.-R. HAMANN \\ Universität Potsdam \\ Am Neuen Palais 10, D-14469 Potsdam, Germany
}

\section{Introduction}

Wolf-Rayet (short: WR) stars are characterized by the bright and broad emission lines which dominate their spectra. This class was originally established for Pop. I stars, distinguishing a nitrogen (WN) and carbon (WC) sequence according to the dominating lines. Wolf-Rayet (specifically, WC) type spectra are also shown by a considerable fraction of central stars of planetary nebulae.

Wolf-Rayet spectra of the carbon subclass are arranged in a subtype sequence from $\mathrm{WC} 2$ to $\mathrm{WC12}$, considering the strength of selected carbon and, as secondary criteria, oxygen emission lines (van der Hucht et al. 1981, Méndez \& Niëmela 1982, Hu \& Bibo 1990). Subtype numbers up to WC6 are comprehensively termed as "early" (short: WCE), the rest as "late" (WCL). We follow van der Hucht et al. 1981 in typing the spectral classification of central stars in brackets.

\section{Model atmospheres for Wolf-Rayet stars}

Radiative transfer calculations for WR atmospheres must account for the extreme non-LTE situation, spherical geometry and the velocity field. Adequate models have been developed during the last years by Hillier (see e.g. Hillier 1990) and by the "Kiel group" (now in Potsdam; cf. Hamann \& Wessolowski 1990). The so-called standard model is based on the idealizing assumptions of spherical symmetry, stationarity and homogeneity. The velocity field is pre-specified (" $\beta$ law"). Present models can handle very complex model atoms (e.g. He, C, N, O) with together a few hundred energy levels and more than thousand explicitely treated line transitions. 


\section{W.-R. Hamann}

These models have been used with some success to analyze Pop. I WR stars. Here we report about their application to WR-type central stars.

It is important to realize than any spectral analysis yields only a combined information about the radius and the mass-loss rate. As a purely spectroscopic parameter we define the "transformed radius"

$$
R_{\mathrm{t}}=R_{*}\left(\frac{v_{\infty} / 2500 \mathrm{~km} \mathrm{~s}^{-1}}{\dot{M} / 10^{-4} M_{\odot} \mathrm{yr}^{-1}}\right)^{2 / 3}
$$

which is independent from the adopted distance of the star and thus plays in expanding atmospheres the role of $\log g$ in the static case. In order to determine the dimensions of the star (i.e. $R_{*}$, which then implies $\dot{M}$ and $L$ ) one of these absolute parameters must be determined independently. For most of the studied [WC] stars the distance is not known. In these cases we set the luminosity to $10^{3.7} L_{\odot}$ as a typical value favored from evolutionary arguments. If there is any idea about the distance of a specific star, $R_{*}, M$ and $L$ may be readily re-scaled using Eq. (1) while keeping $R_{\mathrm{t}}$ constant.

\section{Central stars of subclass [WCE]}

Most CSPN with early-type [WC] spectra are of [WC3] or [WC4] subtype, while [WC5] and [WC6] are very rare. This statistical distribution differs from the Pop. I WC stars, where just the intermediate subtypes (WC5-7) are most frequent. All together twelve [WCE] stars have been analyzed so far by means of detailed non-LTE models (Koesterke \& Hamann 1997 and these proceedings). The stellar temperatures $T_{*}$ (defined as effective temperature related to the stellar radius at Rosseland optical depth 20) ranges from $141 \mathrm{kK}$ (for typical [WC2]) to $126 \mathrm{kK}$ (typical [WC4]), while the mass-loss rates are around $\dot{M}=10^{-6.3} M_{\odot} /$ yr. The wind velocities lie between 3500 and $1200 \mathrm{~km} / \mathrm{s}$. The atmospheric composition is dominated by carbon and helium with a typical mass ratio 1:2, while oxygen is also enhanced (about 10\%).

\section{Central stars of subclass [WC]-PG 1159}

This spectral subclass, which is alternatively termed Of-WR(C) after Méndez (1991), shows weak mass-loss features but otherwise resembles the very hot PG 1159 stars composed mainly of carbon, oxygen and helium (cf. Dreizler et al. 1995). Only a handful of [WC]-PG 1159 stars was known so far, but Acker et al. (these proceedings) now report the detection of many more candidates, putting these stars into her "wels" (weak-emission line stars) category. 
Only three [WC]-PG 1159 stars have been analyzed so far (Abell 30: Leuenhagen et al. 1993; Abell 78: Werner \& Koesterke 1992; Longmore 4: Werner et al. 1993). The few detectable wind lines were analyzed with Wolf-Rayet models, while the weak photospheric absorption profiles could be fitted with static, plane-parallel non-LTE calculations. The latter yield a surface gravity of $\log g=5.5$ [cgs units] in all three cases. Effective temperatures are slightly lower than found for [WCE] stars $(115 \ldots 120 \mathrm{kK})$. The mass-loss rates are lower by a factor of ten, typically (i.e. $\dot{M} \approx 10^{-7.3} M_{\odot} /$ yr), while the expansion velocities are extremely high $(\approx 4000 \mathrm{~km} / \mathrm{s})$. The atmospheres consist of about equal mass fractions of carbon and helium, which is also the typical result for PG 1159 stars.

A close relation between these classes of objects is also suggested by the spectrum variability of Longmore 4 . This object, normally a PG 1159 star, was fortuitously observed in January 1992 when showing a [WC]-PG 1159 spectrum (Werner et al. 1992). These emissions declined within a few days, and the star reached its normal state after two weeks. The mass-loss rate for Longmore 4 during the outburst is estimated to $\dot{M}=10^{-7.3} M_{\odot} / \mathrm{yr}$, while in the quiet state it is lower by at least a factor of five.

\section{Central stars of subclass [WCL]}

A variety of [WCL] stars have been analyzed recently (Leuenhagen et al. 1996; Leuenhagen, these proceedings; DeMarco et al., these proceeding; Leuenhagen \& Hamann 1994). The effective temperatures $T_{*}$ form a sequence correlated with the subtype number, stretching from about $100 \mathrm{kK}$ ([WC7]) to as low as $20 \mathrm{kK}$ ([WC12]). The wind velocities are strongly correlated with the stellar temperature and lie between 1000 and $160 \mathrm{~km} / \mathrm{s}$.

The stellar distance which is needed to determine $L, R_{*}$ and $\dot{M}$ (cf. Sect. 2) is known in some cases (e.g. for MA 17 because it belongs to the $\mathrm{LMC}$, and for $\mathrm{BD}+30^{\circ} 3639$ from the measured angular growth of its nebula). Otherwise again $\log L / L_{\odot}=3.7$ is assumed. The obtained mass-loss rates for the [WCL] stars are of the order of $10^{-6} M_{\odot} \mathrm{yr}^{-1}$. The chemical composition is similar to the PG 1159 stars (roughly equal mass fractions of carbon and helium, and some oxygen).

As a strange result, hydrogen is detected in several [WCL] atmospheres (which is difficult to establish because of the blending nebular hydrogen and stellar He II lines). Nitrogen, otherwise depleted in WC stars, is remarkably strong in the same stars (Leuenhagen, these proceedings). The occurrence of these elements in otherwise helium-burned material requires special scenarios for an explanation. 


\section{Central stars of subclass $[\mathrm{WN}]$ ?}

Central stars of planetary nebula showing a Wolf-Rayet spectrum are always of the WC type. The only exception known yet is the LMC object N66. As a further peculiarity, this central star developed his WN-type spectrum only recently since the year 1990 (Peña et al. 1994, 1995, and these proceedings). Preliminary results of the spectral analysis confirm the helium-rich, hydrogen-deficient composition and yield a rather high effective temperature $\left(T_{*} \approx 100 \mathrm{kK}\right)$.

\section{Discussion}

The "transformed radii" $\log R_{\mathrm{t}}$ (cf. Sect. 2) of all WC-type central stars analyzed so far are plotted versus their effective temperature $\log T_{*}$ in Fig. 1. The [WCL] stars form a nice sequence from low to high temperatures, correlated with a decreasing transformed radius (i.e. increasing wind intensity). At about $120 \mathrm{kK}$ the [WCE] stars form a sequence of roughly constant temperature, but different transformed radii. This sequence is continued, after

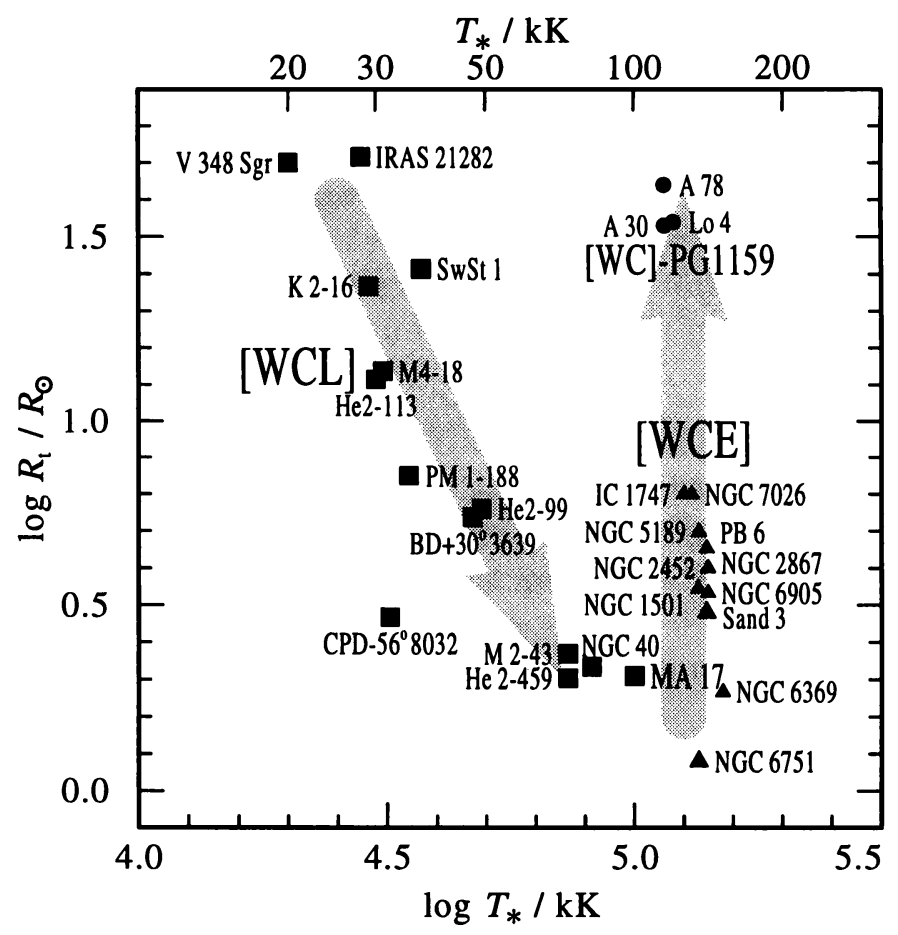

Figure 1. Central stars of Wolf-Rayet type in the $R_{\mathrm{t}}-T_{*}-$ diagram. [WCL], [WCE] and [WC]-PG 1159 subtypes are represented by squares, triangles and circles, respectively. The shades arrows suggest a possible evolutionary connection. 


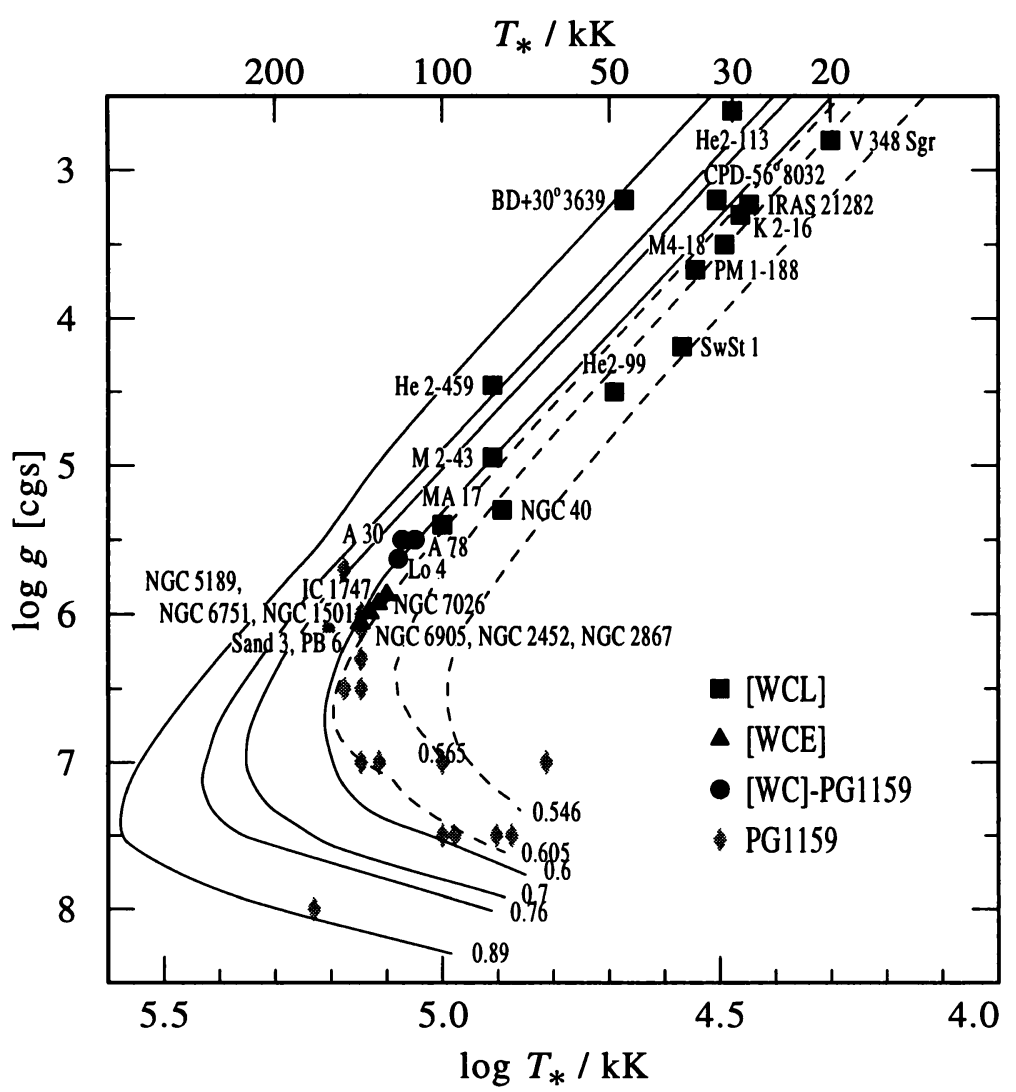

Figure 2. Central stars of Wolf-Rayet type in the $\log g-\log T_{*}$-diagram. [WCL], [WCE] and [WC]-PG 1159 subtypes are represented by squares, triangles and circles, respectively. Shaded diamonds indicate "normal" PG 1159 stars without strong mass-loss (after Dreizler et al. 1995). The tracks for post-AGB evolution taken from Schönberner (1983) or Wood \& Faulkner (1986) (labels: mass in $M_{\odot}$ ) and are only shown for rough comparison; they are not really adequate for the considered objects, as they predict normal surface abundances.

a gap, towards even lower wind intensities (higher $R_{\mathrm{t}}$ ) by the [WC]-PG 1159 subclass. Assuming that all these stars form a continuous evolutionary sequence in that parameter plane, the path indicaied by the shaded arrows is suggested.

In order to visualize possible evolutionary connections we employ the $\log g-\log T_{\text {eff }}$-diagram (Fig. 2). Surface gravities $\log g$ are directly obtained from photospheric absorption lines in the case of [WC]-PG 1159 stars. For the [WCE] and [WCL] stars we take the spectroscopic radii $R_{*}$ and assume a mass of $0.6 M_{\odot}$. "Normal" PG 1159 stars (photospheric spectra analyzed with static model atmospheres) are also shown. The evolutionary tracks included in the figure are not claimed to be adequate in detail for the type 


\section{W.-R. Hamann}

of stars under consideration, but shall indicate roughly an evolution at constant luminosity finally turning into the white dwarf cooling sequence.

This diagram suggests an evolutionary sequence from the [WCL] over the [WCE] stars to the PG 1159 pre-white dwarfs. One problem is posed by the [WC]-PG1159 stars, which appear in Fig. 2 between the [WCL] and the [WCE] class according to their temperature, while Fig. 1 arranges them between the [WCE] and the PG 1159 stars according to the ceasing stellar wind when evolving to higher gravities (possibly crossing an instability, as observed in Lo 4, cf. Sect.4). It is not clear yet whether the effective temperatures are really in that sequence, or if they are subject to systematic errors or the special definition of $T_{*}$.

A second problem with the suggested evolutionary sequence arises from the different chemical composition found for the [WCE] stars (carbon-tohelium $\approx 1: 2$ ) compared to the mass ratio $1: 1$ found for the other types. The significance of this problem must be investigated further.

At present, an evolutionary sequence [WCL] $\rightarrow$ [WCE] $\rightarrow$ [WC]-PG 1159 $\rightarrow$ PG 1159 still appears as a good working hypothesis. However, in case of increasing evidence for the described discrepancies, a more complicated scenario for these different classes of carbon-rich post-AGB stars might be required.

\section{References}

Dreizler S., Werner K., Heber U., 1995, in White Dwarfs, D. Koester and K. Werner (eds.), Lecture Notes in Physics, Springer-Verlag, p. 160

Hamann W.-R., Wessolowski U.: 1990, Astron. Astrophys. 227, 171

Hiller D.J., 1990, Astron. Astrophys. 231, 116

Hu J.Y., Bibo E.A., 1990, Astron. Astrophys. 234, 435

van der Hucht K.A., Conti P.S., Lundstrøm I., Stenholm B., 1981, Space Science Review 28,227

Koesterke L., Hamann W.-R., 1997, Astron. Astrophys. (in press)

Leuenhagen U., Hamann W.-R., 1994, Astron. Astrophys. 283, 567

Leuenhagen U., Koesterke L., Hamann W.-R., 1993, Acta Astronomica 43, 329

Leuenhagen U., Hamann W.-R., Jeffery C.S., 1996, Astron. Astrophys. (submitted)

Méndez R.H., Niëmela V.S., 1982, in Wolf-Rayet Stars, Proc. IAU Symp.99, C.W.H. de Loore \& A.J. Willis (eds.), Reidel, Dordrecht, p. 457

Peña M., Torres-Peimbert S., Peimbert M., Ruiz M.T., Maza J., 1994, Astrophys. J. 428, L9

Peña M., Peimbert M., Torres-Peimbert S., Ruiz M.T., Maza J.: 1995, ApJ 441, 343

Schönberner D., 1983, Astrophys. J. 272, 708

Werner K., Koesterke L., 1992, in The Atmospheres of Early-Type Stars, Lecture Notes in Physics 401, Springer Verlag, p. 288

Werner K., Hamann W.-R., Heber U., Napiwotzki R., Rauch T., Wessolowski U., 1992, Astron. Astrophys. Letters 259, L69

Werner K., Hamann W.-R., Heber U., Napiwotzki R., Rauch T., Wessolowski U., 1993, in Planetary Nebulae, R. Weinberger and A. Acker (eds.), Proc. IAU Symp. No. 155, p. 494

Wood P.R., Faulkner D.J., 1986, Astrophys. J. 307, 659 